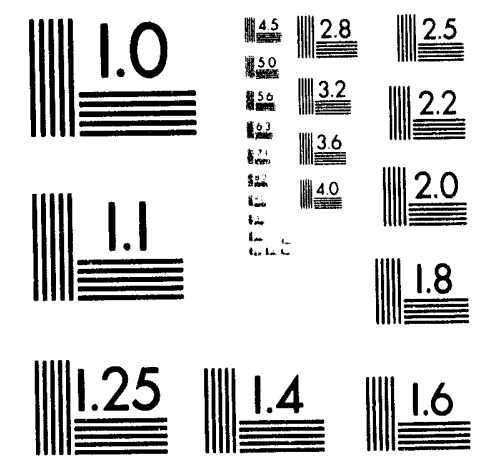



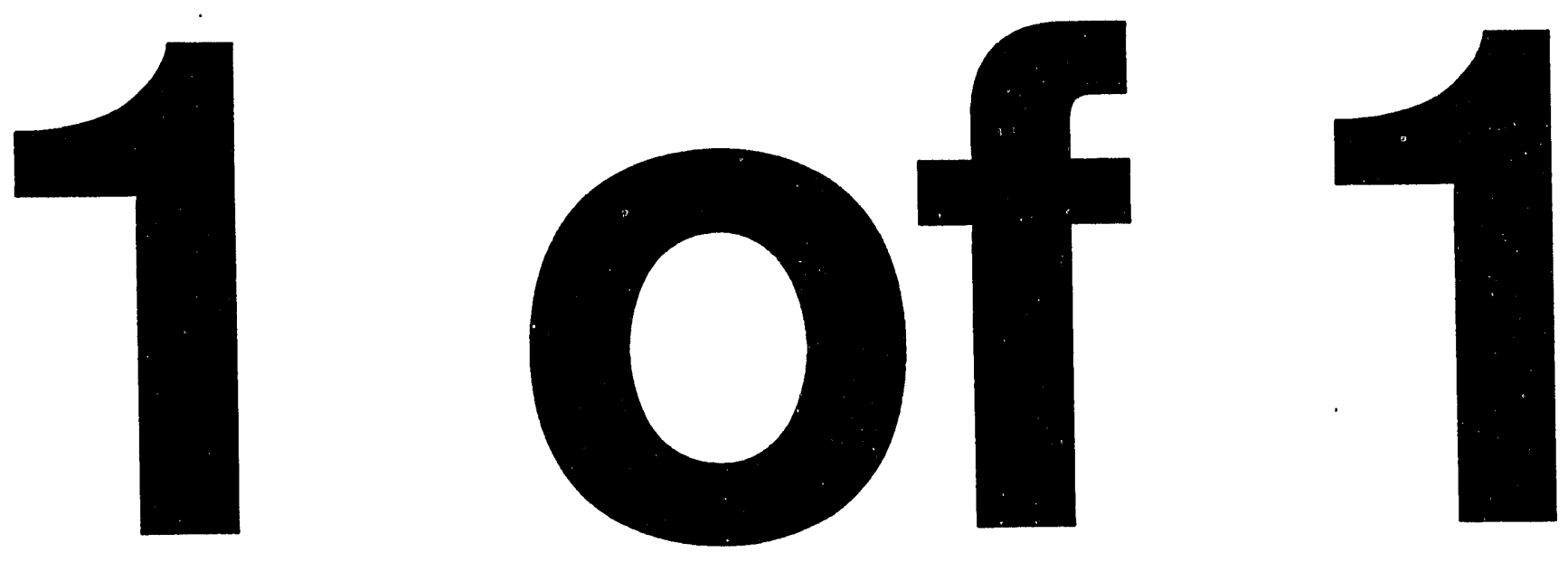


\title{
The Transmission Interface Constraint Problem
}

\author{
Ross Baldick and Edward Kahn \\ Energy \& Environment Division \\ Lawrence Berkeley Laboratory \\ University of California \\ Berkeley, CA 94720
}

October 1993

The work described in this report was funded by the Assistant Secretary of Energy Efficiency and Renewable Energy, Office of Utility Technologies, Office of Energy management Division of the U.S. Department of Energy under Contract No. DE-AC03-76SF00098. 


\section{Table of Contents}

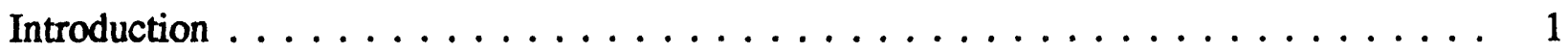

Relationship to Previous Studies $\ldots \ldots \ldots \ldots \ldots \ldots$

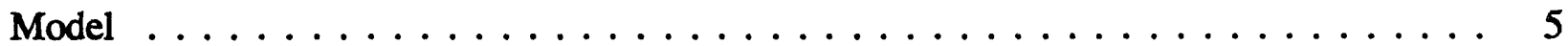

Analysis of Special Cases $\ldots \ldots \ldots \ldots \ldots \ldots$

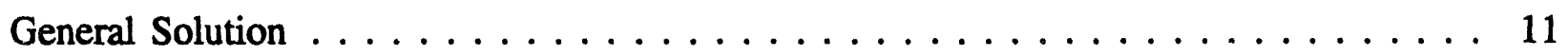

Estimation of the Parameters $\ldots \ldots \ldots \ldots \ldots \ldots$

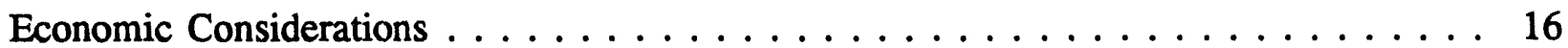

Generalizations and Conclusion $\ldots \ldots \ldots \ldots \ldots$

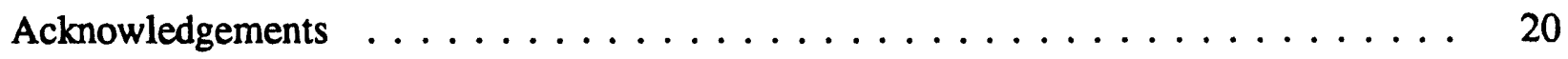

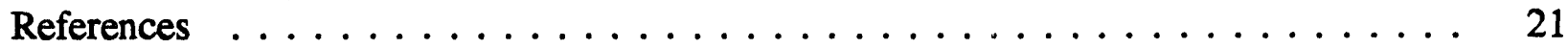

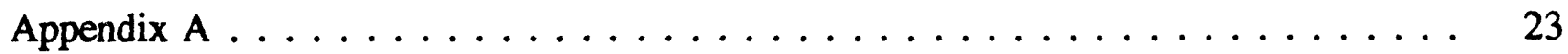




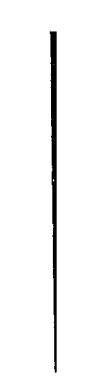

.

i 


\section{List of Tables}

Table 1. Transmission Line Parameters for Individual Circuits in Each Corridor . . . . 14

Table 2. The Value of VArs at $I$ and $L \ldots \ldots \ldots \ldots$ 


\section{List of Figures}

Figure 1. Model System ... . . . . . . . . . . . . . . . . 5

Figure 2. Regions of Optimality for Case A Versus Compensation and Transmission . . 13

Figure 3. Regions of Optimality for Case B Versus Compensation and Transmissions .. 13

Figure 4. Interface Capacity Versus Net Compensation at L $\ldots \ldots \ldots$

Figure 5. Interface Capacity Versus Net Compensation at I . . . . . . . . . . . . . . . . . . . .

Figure 6. Interface Capacity Versus Net Generation at I . . . . . . . . . . . . 15 


\section{Introduction}

Electric power transmission systems exhibit a number of complex constraints on their operation and usage. When a network is subject tc a constraint that limits the amount of power that can be moved from one region to another, there is said to be an interface limit. The power systems literature gives no general treatment of the engineering-economics of this ubiquitous phenomenon. Particular aspects of interface limits are typically discussed in sophisticated technical detail, but the general engineering-economic trade-offs involved in relieving interface constraints have not been systematically addressed. ${ }^{1}$ We approach this problem in the spirit of a heuristic model. Such models are quite valuable under current industry conditions because they delineate technical opportunities and choices in situations where there may be conflicting views among competing parties and regulatory authorities (Baldick and Kahn, 1993a). We organize and enumerate the choices, clarify the practical conditions that dictate the optimum in particular cases, and help to motivate the final choices made by planners.

A working definition of a transmission interface is any set of transmission lines that join one region of a system to another. Typically, there are a few interfaces in a system that are particularly important because the peak flow across each of these interfaces is a significant proportion of the maximum flow that can be reliably supported. The maximum permissible flow depends on the most binding of several constraints on system operation. These constraints include, in normal operation: continuous thermal limits of lines, voltage constraints at buses, steady-state stability limits; and, under contingencies: short-term thermal limits of lines, bus voltage constraints, transient stability limits, limits due to medium-term voltage collapse, and long-term stability limits (Stoll, 1989, Section 17.2). The "normal operating conditions" must, in practice, include provision for outages of lines for scheduled maintenance. Interface limits depend on the load-carrying capabilities of the lines as well as the patterns of flows established across the interface by the generation levels, line impedances and load distributions.

We begin with the representation of transmission line loadability as a function of line length (Dunlop et al., 1979). This representation combines into one curve the constraints due to both voltage and steady state stability criteria for transmission lines operating at various voltages. While thermal limits are relevant for short lines, voltage constraint determine loadability for intermediate lines up to 200 miles in length and stability limits determine loadability for long lines. We focus on the range where the voltage constraint is limiting.

Voltage limits have been referred to recently in a number of separate regulatory settings, both in the United States and internationally, involving lines of length less than 200 miles (APCo, 1991; OFFER, 1992). We will consider only voltage limits and steady-state stability limits in

\footnotetext{
' Standard texts such as Stoll (1989) or Gonen (1988), describe the details of the interface problem, but never give a global analysis of its basic structure.
} 
calculating the transmission interface capacity in a model system. Other issues can be included in a more complete model.

We formulate the problem of serving increased local load without violating an interface constraint. The planning solution is an appropriate trade-off among local generation, transmission upgrades or extensions, and specialized reactive support equipment. While shunt and series compensation is already extensively used in the Western United States, it is less common in the East (EPRI, 1990, p-4). The local generation alternative has been recognized as a generic means of relieving a voltage limited transmission constraint, but there does not appear to be a general treatment of the conditions under which it is economic. ${ }^{2}$ Part of the complexity of the trade-off is the bundled nature of generation service provided at the load center. This service includes not only electric energy, that is, real power, but also voltage support in the form of reactive power.

In Section 2 we describe the relation to previous studies in detail. In Section 3 we model the nature of the interface with reference to a simple system. Although we include several issues in our model such as contingency analysis, we omit most of the details. Our model is therefore illustrative, not definitive. In Section 4 we analyze some special cases of system expansion under the assumption that some of the expansion options are constrained. In Section 5 we analyze the general case, showing that, depending on the values of system parameters, the general problem reduces to one or other of the special cases in Section 4. In Section 6 we evaluate the transmission interface sensitivity parameters for our system, while in Section 7 we make some qualitative observations about the ranges of parameters in typical systems. We also interpret our results as a correction to traditional hierarchical planning criteria based on generation cost only. We conclude in Section 8.

\section{Relationship to Previous Studies}

Planning of electric power systems has historically been performed hierarchically, with generation planned first, then transmission designed to suit the generation, then reactive compensation to satisfy generation and transmission requirements, and then other issues, such as protection and detailed substation layout considered. This hierarchical design is reflected in the technical literature. For example, EPRI (1984b), Lebow et al. (1985), Opoku (1990), and Hong et al. (1990) present formulations of the VAr expansion problem given a fixed plan of generation and transmission expansion, while Stoll (1989, Chapter 16) outlines transmission planning given a fixed plan of generation expansion that is performed based on modeling the system as a single generation and load center as in EPRI (1982a), Bloom et al. (1984), and Stoll (1989, Chapter 14).

\footnotetext{
${ }^{2}$ Mallard (1992, 36-7) addresses this alternative in a cursory fashion in the context of the APCo (1991) Wyoming-Cloverdale transmission project.
} 
EPRI (1987) considers composite generation-transmission expansion, but uses a DC load-flow approximation that does not represent reactive power flows and voltage constraints. EPRI (1988a), formulates the composite generation-transmission problem using an investment cost function that depends, in principle, on both generation and transmission (EPRI, 1988a, Section 3); however, transmission expansion is then handled as a sub-problem with transmission capacities modeled as being independent of generation and compensation (EPRI 1988a, Section 4). ${ }^{3}$

This hierarchical approach suits systems where generation is the dominant cost and where there are limited potential sites for generation expansion, so that transmission and compensation form a relatively small and approximately constant contribution to total costs. This has historically been the case in the United States and continues to be the case in many systems world-wide, particularly in hydro-based systems. The Southern Brazilian electric system, studied in EPRI (1987), for example, has relatively well-defined potential hydro generation sites. These sites necessitate transmission to load centers.

Recently, in the United States, as a greater proportion of new generation is proposed by independent producers, as transmission becomes more difficult to site due to environmental constraints, and as the cost and availability of FACis equipment such as static VAr compensators improves (EPRI 1990, 1991), these historical circumstances are changing (Baldick and Kahn, 1992). For example, repowering options at local sites are likely to compete with proposals for greenfield construction at remote sites. In this case, consideration of transmission and VAr expansion after generation decisions have been made is likely to skew the generation decisions. Distortion of decisions is particularly likely if the costs of transmission and compensation are of the order of magnitude of the difference in costs between local and remote generation. This motivates the incorporation of transmission cost adders into proposed frameworks that evaluate the economics of independent generation proposals (Staschus, et al., 1991).

Some studies consider the generation and transmission systems and reactive power compensation as a composite, but they typically emphasize either reliability/operational issues or long-run generation planning. In the former case, economic trade-offs between generation and transmission investments are typically neglected. In the latter case, transmission constraints are typically reduced to a single cost of capacity representation (Rogers and Rolko, 1992) or approximated as generic adders to generation costs (Head, et al., 1990).

We have modeled a more detailed structure in the transmission and compensation options, but to keep the development clear have used a single-period expansion model as in Rogers and Rolko (1992). Unlike Rogers and Rolko, we have not included the time-variation of demand in our

\footnotetext{
${ }^{3}$ In EPRI (1988b), the analysis allows for information to be fed back from the reactive compensation design sub-problem to the transmission planning sub-problem; however, the authors imply that this happens only in special cases of transmission infeasibility. In our analysis, the effect of compensation on the transmission capacity is much more central.
} 
model. The optimization formulation we develop can be incorporated into a multi-period model with full treatment of load-duration issues as well as lumpiness of construction, albeit with significantly increased computational complexity. While recognizing the importance of these issues, we simplify the analysis for ease of exposition. We also recognize that the feasible increments of transmission capacity are likely to be larger than the increments of generation and, particularly, reactive compensation capacity.

Recent studies of transmission capability enhancement through installation of reactive compensation and phase-shifting transformers model two basic types of transmission configurations: radial transmission from remote generation to load and a complex meshed transmission system (EPRI 1990, 1991). We investigate a network that is conceptually intermediate between radial and highly meshed. It consists of three buses and three lines. We adopt a simplified economic analysis as in EPRI (1991, Sections 3.10 and 5.7), but model the generation expansion options in more detail. Unlike the EPRI studies, however, we have not modeled the underlying low voltage network in the system. As indicated above, the system we investigate is voltage limited, so we did not consider series compensation nor phase-shifters. The effects of these elements could be incorporated into a more comprehensive model.

The study that is closest in spirit to the approach adopted here is Wenyuan and Billinton (1993). These authors consider generation and transmission expansion simultaneously in a framework that represents operating costs, system contingencies, and the customer valuation of outage costs. Their case study reduces to a trade-off similar to the one we address, but the formulation of the reliability constraint differs significantly. We look explicitly at the reactive power aspect of interface limits, while Wenyuan and Billinton confine their assessment to DC load flow relationships and some "normal transmission line capacities." Rather than examine the costs associated with relieving presumed reliability problems, they evaluate alternatives using customer outage costs as a component of total costs. We adopt the opposite approach. Given the very high customer costs of outages, we operate within fixed reliability criteria, and examine the various ways to relieve interface constraints due to reactive power requirements. Again, our framework could be incorporated into Wenyuan and Billinton's Monte Carlo approach to achieve a probabilistic reliability assessment of composite generation and transmission, with a considerable increase in the computational complexity (EPRI, 1982b, IEEE, 1990). Another extension is to consider multi-attributes for optimization instead of a single cost criterion. This approach is described in EPRI (1984a). 


\section{Model}

\section{The System}

For concreteness, we will model the three node system with nodes G, I, L interconnected by three corridors of lines and illustrated in Figure 1. The node $G$ is a center of generation, while $L$ is a load center. Node $I$ is geographically intermediate between $G$ and $L$ and can support some new generation capacity. The cost of new/ generation at $L$ is more expensive than at $I$, which is, in turn, more expensive than generation at $\mathrm{G}$. We assume that the costs of generation are adjusted to reflect outages rates.

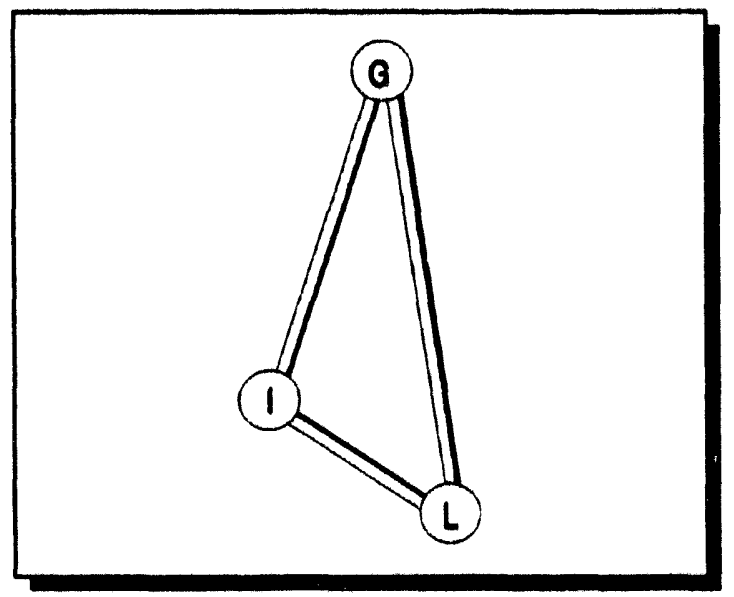

The corridors from $G$ to $I$ and from $I$ to $L$ are strong while the corridor from $G$ to $L$ is weak. By strong and weak, we mean that the transfer capability of the system is primarily limited by the voltage drop across the weak line. In particular, to illustrate the nature of interface constraints, we will calculate the limit on the export of power from $G$ to I and $L$. It will turn out that for the particular line parameters, the $\mathrm{G}$ to $\mathrm{I}$ and $\mathrm{L}$ interface capacity is limited by low voltage criteria at $\mathrm{L}$ under a contingency. ${ }^{4}$

To illustrate the generation-transmission-reactive compensation expansion problem, we will assume that the load at $\mathrm{L}$ is served by the generation at $\mathrm{G}$ and $\mathrm{I}$. There is just enough generation to supply $\mathrm{L}$ reliably. The transmission interface constraint is just binding, so that increased generation at $\mathbf{G}$ would necessitate an increase in the interface capacity. Increased generation at $\mathrm{L}$ would not be bound by this interface constraint, but is more expensive than remote generation at $\mathrm{G}$. Increased compensation at $\mathrm{I}$ or $\mathrm{L}$ as well as increased generation at I can be expected to alter the interface capacity.

We assume that new load growth is expected at L, requiring new generation at either G, I, or $\mathrm{L}$, or some combination of the three. Transmission construction in the $\mathrm{G}$ to $\mathrm{L}$ corridor and reactive compensation at $\mathrm{I}$ or $\mathrm{L}$ is also possible.

\footnotetext{
4 This simple triangular geometry roughly represents the system analyzed in detail in APCo (1991) under the following equivalences: the load center $\mathrm{L}$ corresponds to the Matt Funk and Cloverdale stations; the generation node $G$ corresponds to the stations at Baker, Culloden, Amos and Kanawha River; and the intermediate node corresponds to the Broadford and Jacksons Ferry stations. The binding constraints in this case included low voltage levels under contingencies.
} 


\section{Symbols}

We define the following symbols:

$\Delta L$ is the increase in real power load at $\mathrm{L}(\mathrm{MW})$. We assume that the angle between current and voltage for this load is $\phi$, so that the power factor is $\cos \phi$ and the increase in load reactive power is $\Delta L \tan \phi$.

$P_{G}, P_{I}, P_{L}$ are the increased generations installed and on-line at $G, I$, and $L$ to supply the incremental load at $\mathrm{L}(\mathrm{MW})$. Reactive power production capacity comes bundled with the real power generation capacity. We assume that at the rated generation level, the minimum power factor of the generators is $\cos \theta$, so that the reactive power productions satisfy: $Q_{G} \leq P_{G} \tan \theta, Q_{I} \leq P_{I} \tan \theta, Q_{L} \leq P_{L} \tan \theta$, respectively.

$C_{G}, C_{l}, C_{L}$ are the costs of generation expansion at $G, I, L(\$ / M W)$, including local substation upgrades. We assume that:

1) $C_{G}<C_{I}<C_{L}$, so that local generation is more expensive than remote generation;

2) costs include derating of capacity to represent outage rates, so that we can consider the capacity increments to be reliable capacity increments; and,

3) energy costs have been incorporated into the capacity

costs. $\Delta T$ is the effective increase in the reliable transmission interface capacity due to transmission construction (MW). We think of this capacity as being built in the $\mathrm{G}$ to $\mathrm{L}$ corridor because this is the limiting corridor. Notice, however, that $\Delta T$ may not be equal to the thermal capacity of the new transmission.

$C_{T}$ is the effective cost of increasing the transmission interface capacity (\$/MW), including substation upgrades.

$Q_{C}$ is the increase in capacitive compensation at L (MVAr).

$C_{C}$ is the cost of compensation (\$/MVAr), including substation upgrades. We assume that $C_{C}<C_{G}$; however, $C_{C}$ may be a significant proportion of $C_{L}-C_{G}$.

$\eta, v$ are the sensitivities of the interface limit to increased reactive power production at $L$ and I, respectively (MW/MVAr). The reactive power can be provided either by compensation equipment or from generators. These parameters are calculated in Section 6 for the example system, given some particular assumptions on line impedances and voltage limits. In general, the parameters will vary with the level of compensation. Stoll (1989, p753-4) gives an example where $\eta$ is approximately one-third to one-half. We assume that $\eta>v$ since compensation at $\mathrm{L}$ is more effective in relieving the voltage constraint at $\mathrm{L}$ than compensation elsewhere. Therefore, reactive compensation will never be added at $I$ in preference to compensation at $L$.

$\epsilon$ is the sensitivity of the interface limit to increased real power production at $I(M W / M W)$. In the example system, which is voltage limited, $\epsilon$ will turn out to be negative, but in other 
systems, particularly stability limited systems, it can be positive. In a stability limited system, we would expect that local generation at $\mathrm{L}$ would also affect the interface capacity; however, in our voltage limited system we will assume that increases in local real generation do not directly affect the interface capacity.

\section{Limitations and Assumptions}

We will neglect changes in the average percentages of real and reactive losses due to changes in flows. Fixed percentage losses in transmission lines are assumed to be incorporated as adders in the generation costs. While the reduction in percentage real losses is often a significant aspect of the benefits of a new line, we will assume that percentage losses are also approximately independent of the particular capacity expansion undertaken. 5 Changes in percentage real losses can be incorporated into a more complete analysis.

We will consider increases in interface capacity due to reactive generation by new generation installed at $\mathrm{I}$. In doing so, we are assuming that generation at $\mathrm{I}$ is on-line and available whenever the increased interface capacity is required to support peak and shoulder demand at L. Since operating costs at I are likely to be higher than operating costs at G, this may appear to be "out-of-merit" dispatch if transmission constraints are ignored. However, when the transmission constraints are included, the value of voltage support at I justifies having I on-line (OFFER, 1992, Kahn, 1993).

In practice there are limits to the relief of the interface constraint as we increase compensation and as we increase generation at $L$ or $I$. This appears as a reduction in the sensitivities $\eta, v$. However, they will be modeled as constants. We will also assume that they are the same for the existing network and for the network with expansion in the $\mathrm{G}$ to $\mathrm{L}$ corridor. We would expect the sensitivity to increase after the addition of any transmission line. Variation of $\eta, v$ and $\epsilon$ with levels of compensation and with levels of transmission construction can be incorporated into a more sophisticated non-linear model.

We will assume that new transmission is fully reactively compensated and that the costs of compensation are incorporated into the cost of transmission. We neglect lumpiness in transmission and generation construction, which in practice can be a significant factor. (For a discussion of lumpiness in transmission construction, see Baldick and Kahn, (1993b) and EPRI (1987, Section 6.6).)

\footnotetext{
${ }^{5}$ Loss savings were calculated in APCo (1991), but were not used in the justification of the line. Mallard (1992) analyzes the value of loss reductions for that case.
} 


\section{Constraints and Formulation}

The real load at $\mathrm{L}$ must be supplied by one of the three generators. Therefore: $\Delta L=P_{G}+P_{I}+P_{L}$. The reactive load at $L$ must by supplied by one of the generators or by added compensation at $\mathrm{L}$. We assume that additional transmission is fully compensated so that it neither increases nor decreases the supply of reactive power in the system. Therefore: $\Delta L \tan \phi \leq\left(P_{G}+P_{I}+P_{L}\right) \tan \theta+Q_{C}$. We will assume that $\tan \phi \leq \tan \theta$ so that this constraint on reactive power supply is never binding. The generation increase at $G$ is limited by the increase in the interface capacity. The interface capacity can be increased in three ways: by construction of new transmission capacity; by increased compensation in the system; or, by the increased reactive generation made available by increased generation capacity at $L$ or $I$. However, the interface capacity is correspondingly decreased by net reactive load at $\mathrm{L}$. The interface capacity is also modified by increased generation levels at $\mathrm{I}$. That is, we assume that the interface constraint can be represented by the linear inequality ${ }^{6}$ : $P_{G} \leq \Delta T+\eta\left(Q_{C}+Q_{L}-\Delta L \tan \phi\right)+v Q_{I}+\epsilon P_{I}$. Since $Q_{I}, Q_{L}$ are bounded in terms of $P_{I}, P_{L}$, respectively, we can rewrite this as: $P_{G} \leq \Delta T+\eta\left(Q_{C}+P_{L} \tan \theta-\Delta L \tan \phi\right)+(v \tan \theta+\epsilon) P_{r}$. We will assume that $v \tan \theta+\epsilon \geq 0$, so that the net effect of generation at $I$ is to relieve the interface constraint. In general, the interface capacity can only be increased until stability and thermal limits become binding. To incorporate maximum limits on the increase in the interface capacity that are possible from compensation and local generation, we must define an additional variable $\Delta T_{C}$, w r i t e $P_{G} \leq \Delta T+\Delta T_{C}$, and add the constraints: $\Delta T_{C} \leq \Delta T_{C}^{\max }, \Delta T_{C} \leq \eta\left(Q_{C}+P_{L} \tan \theta-\Delta L \tan \phi\right)+(v \tan \theta+\epsilon) P_{I}$.

The total costs of construction are: $C_{G} P_{G}+C_{I} P_{I}+C_{L} P_{L}+C_{C} Q_{C}+C_{T} \Delta T$. The optimal expansion plan minimizes the costs subject to the constraints. This is a linear program.

\footnotetext{
${ }^{6}$ We are assuming that the interface capacity is primarily constrained by voltages due to one particular outage. This is the case in our example system, except for high levels of uncompensated generation at I. For our choice of parameters, the critical contingency is almost always in the $G$ to $I$ corridor. In general, we need a constraint for each contingency. Each linearized constraint will have corresponding coefficients $\eta, v, \epsilon$. For any particular combination of generation and compensation, one of the constraints will be most binding.
} 


\section{$4 \quad$ Analysis of Special Cases}

Before analyzing the general case, we consider some special cases that can be solved easily. Each special case involves the assumption that some of the expansion options are constrained a priori to be zero. In Section 5 we will remove these assumptions by showing that each of the assumed constraints on options coincides with optimal expansion for some combination of the cost parameters.

Case 1. $P_{G}=0, P_{I}=0, Q_{C}=0, \Delta T=0$. In this case, the only option is increased generation at $\mathrm{L}$. We have: $P_{L}=\Delta L$. The total cost is $\Delta L C_{L}$ for a capacity cost of $C_{L^{\circ}}$

Case 2. $P_{L}=0, P_{I}=0, Q_{C}=0$. In this case, the only option is increased generation at $G$ and commensurate transmission construction to accommodate the increased real and reactive power flows. We have: $P_{G}=\Delta L, P_{G} \leq \Delta T-\eta \Delta L \tan \phi$, so for minimum costs: $\Delta T=\Delta L(1+\eta \tan \phi)$. The total cost is $\Delta L\left(C_{G}+(1+\eta \tan \phi) C_{T}\right)$ for a capacity cost of $C_{G}+(1+\eta \tan \phi) C_{T}$. This cost is larger than $C_{G^{*}}$ by $(1+\eta \tan \phi) C_{T}$. which we can refer to as the effective cost of transmission to relieve the interface constraint. This option is attractive if the effective cost of transmission is less than the difference in cost between generation at $\mathbf{G}$ and generation at $I$ or $L$. This may be the case if:

a) there is considerable excess generation at $G$, so that the cost of generation at $G$ is essentially just energy costs, or,

b) the cost of generation at $\mathrm{I}$ and $\mathrm{L}$ is large due to siting considerations.

The first possibility corresponds to the situation described in APCo (1991, Sections IV-VI). The constraint $Q_{C}=0$ reflects the fact that in the APCo case compensation had already been added to relieve the interface constraint up to the limit $\Delta T_{C}^{\max }$ (see APCo, 1991, IV-4). The addition of such local compensation is represented in our next special case.

Case 3. $P_{L}=0, P_{I}=0, \Delta T=0$. In this case, we must add compensation to the line to support increased generation at $G$. The cost is $C_{G} P_{G}+C_{C} Q_{C}$, where $P_{G}=\Delta L, P_{G} \leq \eta\left(Q_{C}-\Delta L \tan \phi\right)$, so for minimum cost, $Q_{C}=\Delta L((1 / \eta)+\tan \phi)$. Therefore, the total expansion cost is equal to $\Delta L\left(C_{G}+((1 / \eta)+\tan \phi) C_{C}\right)$, for a capacity 
cost of $C_{G}+((1 / \eta)+\tan \phi) C_{C}$. This cost is larger than $C_{G}$ by $((1 / \eta)+\tan \phi) C_{C}$, which we can refer to as the effective cost of using reactive power support to relieve the interface constraint. This option is attractive if this effective cost is less than the effective transmission construction costs and is also less than the difference between generation costs at $G$ and at $I$ or $L$. The effective cost of relieving the interface limit depends very sensitively on $\eta$.

Case 4. $P_{I}=0, \Delta T=0, Q_{C}=0$. In this case, $\Delta L=P_{G}+P_{L}$. The addition of generation at $\mathrm{L}$ provides reactive power that relieves the interface constraint, allowing increased generation at $G$. That is, $P_{G} \leq \eta\left(P_{L} \tan \theta-\Delta L \tan \phi\right)$. Because the cost of generation at $\mathrm{L}$ is higher than that at $\mathrm{G}$, this constraint will be binding at the optimum solution, so: $P_{G}=\eta\left(P_{L} \tan \theta-\Delta L \tan \phi\right)$.

Therefore, $\quad P_{L}=\Delta L \frac{1+\eta \tan \phi}{1+\eta \tan \theta}, P_{G}=\Delta L \eta \frac{\tan \theta-\tan \phi}{1+\eta \tan \theta}$, for a total cost of $\Delta L \frac{(1+\eta \tan \phi) C_{L}+\eta(\tan \theta-\tan \phi) C_{G}}{1+\eta \tan \theta}$, a nd a c a p c it y cost of $\frac{(1+\eta \tan \phi) C_{L}+\eta(\tan \theta-\tan \phi) C_{G}}{1+\eta \tan \theta}$. This cost is greater than $C_{G}$ by the amount $\frac{1+\eta \tan \phi}{1+\eta \tan \theta}\left(C_{L}-C_{G}\right)$, which we can again refer to as an effective cost of relieving the transmission interface constraint. In this case, the effective cost of relieving the interface constraint is much less sensitive to changes in $\eta$ as compared to the last case. The capacity cost is smaller than $C_{L}$ so long as $\tan \theta>\tan \phi$.

Case 5. $P_{L}=0, Q_{C}=0, \Delta T=0$. In this case, the options are increased generation at $G$ and I. We have: $P_{G}+P_{I}=\Delta L$. As in the last case, reactive generation at $I$ relieves the interface constraint, allowing increased generation at $G$. That is, $P_{G} \leq(v \tan \theta+\epsilon) P_{I}-\eta \Delta L \tan \phi$. Because the cost of generation at $I$ is higher than that at $G$, this constraint will be binding at the optimum solution, so:

$P_{G}=(v \tan \theta+\epsilon) P_{I}-\eta \Delta L \tan \phi$. 
Therefore, $P_{I}=\Delta L \frac{1+\eta \tan \phi}{1+v \tan \theta+\epsilon}, P_{G}=\Delta L \frac{v \tan \theta+\epsilon-\eta \tan \phi}{1+v \tan \theta+\epsilon}$, for a total cost of $\Delta L \frac{(1+\eta \tan \phi) C_{I}+(v \tan \theta+\epsilon-\eta \tan \phi) C_{G}}{1+v \tan \theta+\epsilon}$, and a capacity cost of $\frac{(1+\eta \tan \phi) C_{I}+(v \tan \theta+\epsilon-\eta \tan \phi) C_{G}}{1+v \tan \theta+\epsilon}$. This cost is greater than $C_{G}$ by the amount $\frac{1+\eta \tan \phi}{1+v \tan \theta+\epsilon}\left(C_{I}-C_{G}\right)$, which we can again refer to as an effective cost of relieving the transmission interface constraint. As in case 4 , the effective cost of relieving the interface constraint is much less sensitive to changes in $\eta$ as compared to case 3 . The capacity cost is smaller than $C_{I}$ so long as $v \tan \theta+\epsilon>\eta \tan \phi$.

The attraction of the last two cases is that by installing some capacity at $L$ or I, the increased interface limit allows cheaper capacity to be installed at $G$ without transmission expansion. The option may be attractive if a generator at $\mathrm{L}$ or I could supply a significant amount of reactive power in excess of the local incremental reactive power demand; that is, if $\tan \theta$ is particularly large for this generator compared to $\tan \phi$.

Some contracts for independent power require power factors of $85 \%$, which corresponds to $\tan \theta=62 \%$. One example of this kind is the Hopewell project, now operating in Virginia (Hopewell, 1988). If $\tan \theta$ is significantly larger than $\tan \phi$ because of, say, reactive compensation of the incremental load at the distribution system level, then the addition of generation at $\mathrm{L}$ can allow for a significant proportion of the total generation to be supplied from G. If capacity at $L$ is only marginally more expensive than capacity at $I$, then this may be an optimal plan even if we remove the assumption of no generation at $I$. We will see precise conditions for this case in the next section.

\section{General Solution}

For the simple system and our linear model, we can derive analytically the conditions under which various expansion options are optimal.

Fact: First assume that there is no limit on the increase in the interface capacity available from compensation and local generation. Then, remote generation is part of the optimal expansion plan. To support this generation, there must be either transmission, local compensation, or generation at $I$ or $L$ to relieve the interface constraint. There are two main cases. 
Case A: If $\frac{1}{1+v \tan \theta+\epsilon}\left(C_{I}-C_{G}\right)<\frac{1}{1+\eta \tan \theta}\left(C_{L}-C_{G}\right)$ then:

(i): if $\frac{1}{\eta} C_{C}<\frac{1}{1+v \tan \theta+\epsilon}\left(C_{I}-C_{G}\right)$ or $C_{T}<\frac{1}{1+v \tan \theta+\epsilon}\left(C_{I}-C_{G}\right)$ then generation at $G$ and either local compensation or transmission is optimal, depending on whether $C_{C} / \eta$ or $C_{T}$ is smaller,

(ii): otherwise, generation at $\mathrm{G}$ and $\mathrm{I}$ is optimal.

Case B: If $\frac{1}{1+v \tan \theta+\epsilon}\left(C_{I}-C_{G}\right) \geq \frac{1}{1+\eta \tan \theta}\left(C_{L}-C_{G}\right)$ then:

(i): if $\frac{1}{\eta} C_{C}<\frac{1}{1+\eta \tan \theta}\left(C_{L}-C_{G}\right)$ or $C_{T}<\frac{1}{1+\eta \tan \theta}\left(C_{L}-C_{G}\right)$ then generation at $\mathrm{G}$ and either local compensation or transmission is optimal, depending on whether $C_{C} / \eta$ or $C_{T}$ is smaller,

(ii): otherwise, generation at $G$ and $L$ is optimal.

The amount of generation at $\mathrm{G}, \mathrm{I}$, and $\mathrm{L}$, the amount of compensation and transmission, and the cost of the optimal plan can be determined from the analyses of special cases in Section 4.

If there is a limit on relieving the interface constraint, then build according to the above prescription until the limit becomes binding. Then build either more remote generation and transmission or build generation at $\mathrm{I}$, depending on whether $C_{T}$ or $C_{I}-C_{G}$ is smaller. Again, the cost can be determined from the cases analyzed in Section 4.

Proof: See Appendix A. 
Figure 2. Regions of Optimality for Case A Versus Compensation and Transmission

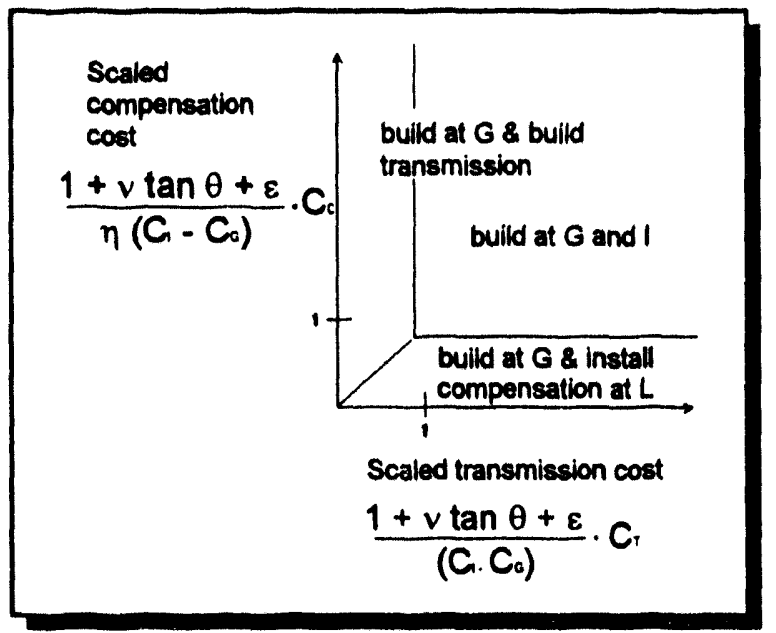

Figure 3. Regions of Optimality for Case B Versus Compensation and Transmissions

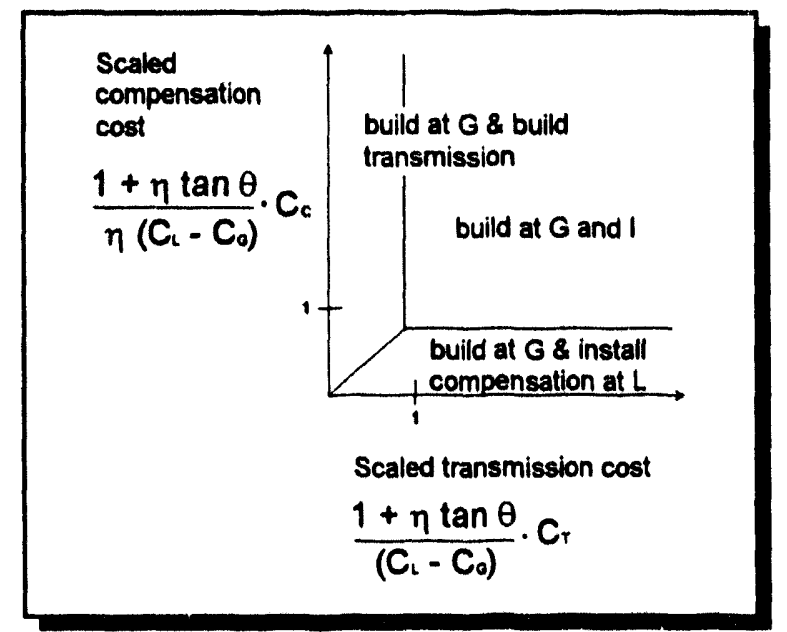

Cases $A$ and $B$ are illustrated in Figures 2 and 3, where the regions of optimality are plotted versus scaled costs. For each set of values of the parameters, the optimal construction strategy is shown, assuming that any limit on the increase in interface capacity is not binding. In these cases the optimal strategy involves construction of two options. If the limit on the increase in the interface capacity is binding, then three or more options will be required in the optimal plan.

Qualitatively, and consistent with expectation, at low relative costs of transmission or compensation, the optimal plan involves remote generation at $\mathrm{G}$ supported by transmission or compensation at $\mathrm{L}$, respectively. As relative costs of transmission and compensation increase, the optimal plan shifts towards either generation at $G$ and $I$ or generation at $G$ and $L$, depending on the relative cost of generation at $I$. Since the difference between costs at $G$ and $L$ or $I$ can be expected to vary widely depending on circumstances, it is necessary to have estimates of the cost of transmission and reactive compensation to determine the most economical expansion. In the next two sections, we evaluate $\eta, v$, and $\epsilon$ for an example system.

\section{Estimation of the Parameters $\eta, v, \epsilon$}

Evaluation of $\eta, v, \epsilon$ depends on case particulars including transmission line characteristics and the form of the reliability criterion. We provide an illustrative example. The $G$ to $I$ and I to $\mathrm{L}$ corridors consist of pairs of $765 \mathrm{kV}$ lines of lengths 125 and 50 miles, respectively, while the $\mathrm{G}$ to $\mathrm{L}$ corridor consists of pairs of $345 \mathrm{kV}$ lines of length 150 miles. Line data is taken from Stoll (1989, Tables 16.2 and 16.3) and the individual circuits in each corridor have threephase per unit parameters as shown in Table 1. The base is 100 MVA. We have neglected generator, transformer, and termination impedances in our system model, but recognize that these must be considered in a complete analysis (Dunlop et al., 1979). 
Table 1. Transmission Line Parameters for Individual Circuits in Each Corridor

\begin{tabular}{lllll}
$\begin{array}{l}\text { Line in } \\
\text { Corridor }\end{array}$ & $\begin{array}{l}\text { Series } \\
\text { Resistance }\end{array}$ & $\begin{array}{l}\text { Inductive } \\
\text { Reactance }\end{array}$ & $\begin{array}{l}\text { Shunt } \\
\text { Capacitance }\end{array}$ & $\begin{array}{l}\text { Thermal } \\
\text { Capacity }\end{array}$ \\
\hline$G-1$ & 0.00044 & 0.0114 & 5.9 & 70.2 \\
$G-L$ & 0.0076 & 0.076 & 1.3 & 11.9 \\
I-L & 0.00018 & 0.0046 & 2.35 & 70.2 \\
\hline
\end{tabular}

All impedanoes in per unit on a 100 MVA base.

Source: data is taken from Stoll (1989, Tables 16.2 and 16.3)

This choice of model and parameters is based on our stylized characterization of the existing network surrounding APCo's Wyoming-Cloverdale project. Of course, our simple triangular network does not represent the full complexity of the real configuration and results based on it are therefore illustrative rather than definitive.

The lengths of the lines suggests that the binding constraints on interface capacity will be due to voltage and steady-state stability. We will discuss in detail the voltage and steady-state stability constraints, but will neglect the transient stability and other constraints. We also ensured that thermal constraints were not violated.

Under normal operation, all lines are in service. We do not consider scheduled maintenance of lines, although this is important in a complete analysis. The voltage criterion for normal operation is that with generator voltages as high as possible, but not exceeding 1.00 per unit, all bus voltages should be between 0.97 and 1.03 per unit. The steady-state stability criterion is that the maximum angle across the network should be less than 40 degrees.

The voltage criterion for contingency cases is as follows. After any single line outage and with generator voltages as high as possible, but not exceeding 1.00 per unit, the voltage at a bus in the system will not fall below 0.95 per unit. Ramping constraints on change of reactive power production by generators are neglected. The steady-state stability criterion is that the maximum angle across the network after the contingency should be less than 40 degrees.

These criteria are typical of voltage and steady state stability criteria in industry use; however, individual utilities will have different particulars. We found various combinations of load real and reactive power and generation at I that just satisfied all the constraints. In each case, the binding constraint was the voltage at $L$ after loss of one of the lines in the $G$ to $I$ corridor. We ensured that the voltage was not too high if the outaged line was returned to service.

We investigated the trade-off between compensation and transfer capacity. In each case, we kept the net reactive shunt compensation (that is, load minus compensation) constant, and found the real load power that brought the voltage down to 0.95 per unit. We are implicitly assuming that the compensation can be adjusted in small enough increments as the load varies so that post- 
Figure 4. Interface Capacity Versus Net Compensation at $L$

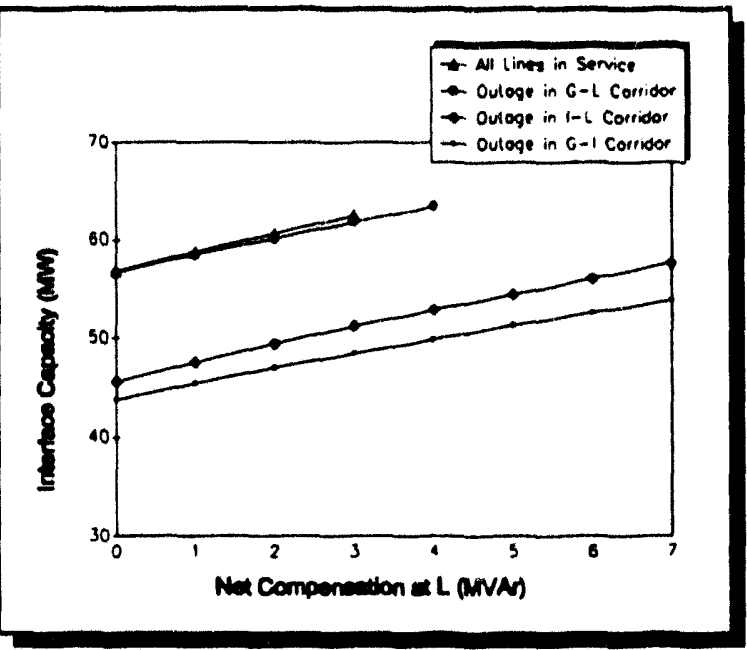

Figure 6. Interface Capacity Versus Net Generation at I

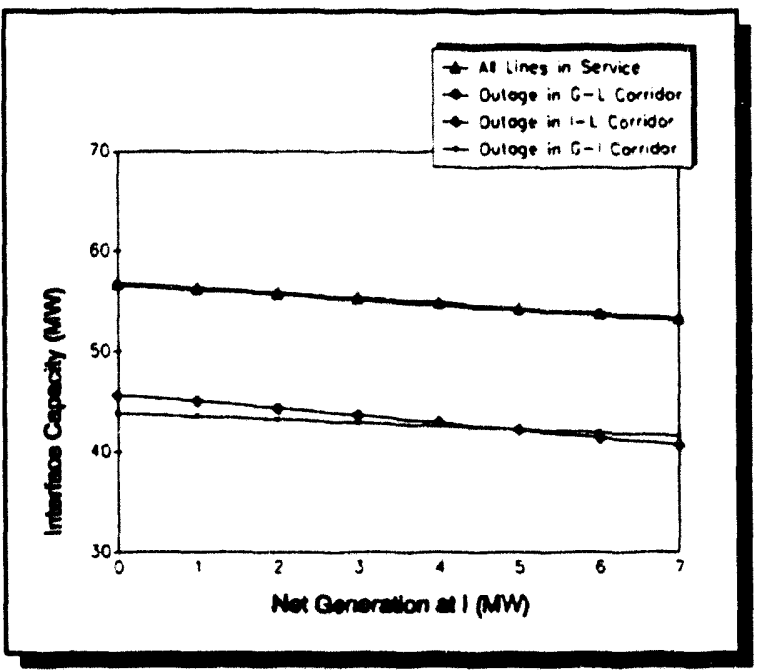

Figure 5. Interface Capacity Versus Net Compensation at I

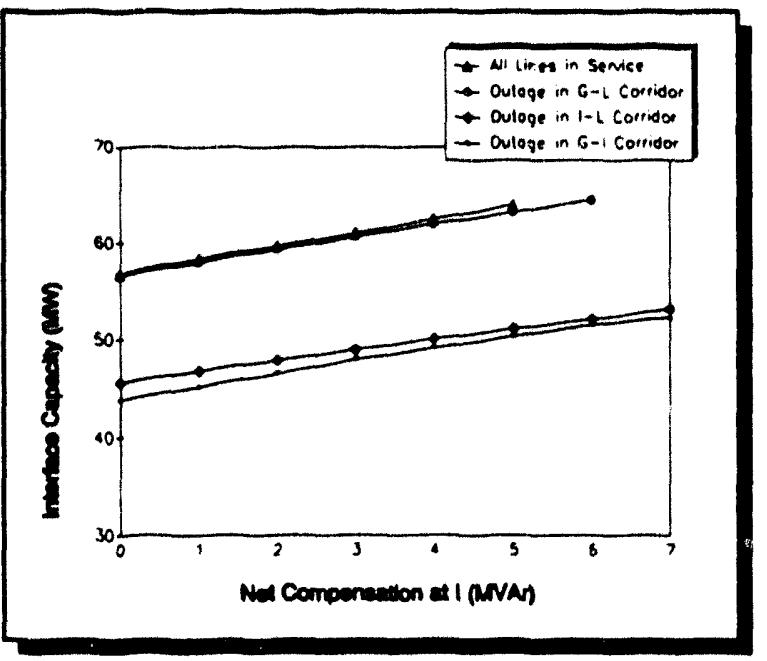

contingency voltages are always within bounds without post-contingency readjustment of compensation.? In particular, we assume that any shunt inductors on the $765 \mathrm{kV}$ system are compensated or can be switched in small steps as load changes, so that high-voltage problems under low load or loss-of-load can be avoided (EPRI, 1988c, Section 4.3, APCo, 1991, p IV$3)$.

Figures 4,5 , and 6 show the results of the study. The graphs show interface capacity (that is, load at $\mathrm{L}$ minus generation at I) versus net compensation at $L$ and $I$ (in figures 4 and 5 ) and versus real generation at I (in Figure 6). In each case, two of these variables were held constant while the other was varied. Initial net generation at I was five per unit. The parameters $\eta, v$, and $\epsilon$ are, respectively, the slopes of the most binding interface capacity curves versus compensation at $L$, compensation at $I$, and generation at $I$. For example, in figure $4, \eta$ is the slope of the curve for the G-I outage. For this system and these reliability criteria, the study

\footnotetext{
${ }^{7}$ As discussed above, we ignore transient stability issues. In practice, these may necessitate that some of the compensation be rapidly and continuously adjustable (EPRI 1991, Section 3.6). We are also assuming that knowledge of net generation and compensation at $I$ and $L$ is sufficient to determine stability. In practice, characteristics of generators and loads will also affect stability.
} 
indicates values of $\eta$ in the range 1.3 to 1.6 and values of $v$ in the range 1.2 to 1.4 for net compensation in the range of zero to seven per unit. We also found values of $\epsilon$ in the range 0.2 to -0.35 for increases in real power generation at $I$ in the range of zero to seven per unit. ${ }^{8}$ In a more realistic design example, including a more complete system model, other considerations may make $\eta$ and $v$ smaller. As indicated above, Stoll (1989) finds for series and shunt compensation in another system values of $\eta$ in the range one-third to one-half. Studies reported in EPRI (1991) result in values of $\eta$ of about 0.8 .

\section{$7 \quad$ Economic Considerations}

The general solution outlined in Section 6 depends upon numerous parameters. In this section we develop some rough estimates of how the optimal choices will work out in representative cases. Order of magnitude estimates of typical cost ranges are introduced to show the sensitivity of results to parameters. We will also give an indication of how issues such as lumpiness change the analysis.

One interpretation of our general result is that it provides a "network externality" correction to the traditional planning criteria and procedures based primarily on comparison of generation costs. The key insight offered by our model is that when either $\mathbf{C}_{\mathbf{I}}-\mathbf{C}_{G}$ or $\mathbf{C}_{\mathbf{L}}-\mathbf{C}_{G}$ is relatively small, there are substantial advantages to building generation at the intermediate node or local nodes, respectively. It is not unreasonable to expect differences in generation cost on the order of $\$ 100-\$ 300 / \mathrm{kW}$, or even more. Such differences could arise where the same technology was used at all sites, but economies or penalties resulted from project scale economies, infrastructure cost, or pollution control requirements. If different technologies are used at the sites $\mathrm{G}, \mathrm{I}$, and $\mathrm{L}$, then cost differences of this magnitude are also not unreasonable. ${ }^{9}$

The advantages of local or intermediate node generation in relieving transmission interface constraints is captured in our model by the network parameters $\eta, \nu$ and $\epsilon$. We illustrate the magnitude of the network externality effects summarized by these parameters and show how

\footnotetext{
We also investigated other systems with the same geometry and differing line parameters. All gave results for $\eta$ in the range of one to two. Other geometries and other binding constraints can be expected to give different values. For net compensation less than zero, the values of $\boldsymbol{\eta}$ and $\mathbf{v}$ were larger. For generation construction at 1 , we are primarily interested in the net effect of simultaneously increased real and reactive generation. We therefore also calculated the change in interface capacity for simultaneous increases in real and reactive generation at $\mathrm{I}$. The results were very close to the linearized approximation.

- For example, Mallard (1992) estimates the present-value cost of cost of a pulverized coal plant operating at $50 \%$ load factor to be $\$ 4160 / \mathrm{kW}$ in 1998 compared to $\$ 4346 / \mathrm{kW}$ for a gas-fired combined cycle plant, for a difference of $\$ 186 / \mathrm{kW}$.
} 
these effects compare with the differences in generation costs. . For convenience the factor $\frac{1}{1+v \tan \theta+\epsilon}$ will be called $V\left(R_{V}\right)$ and the factor $\frac{1}{1+\eta \tan \theta}$ will be called $V\left(R_{L}\right)$. These factors reflect the value of compensation provided by generation at $I$ or $L$. Table 2 summarizes a few cases.

Table 2. The Value of VArs at $I$ and $L$

\begin{tabular}{|c|c|c|c|c|c|c|}
\hline $\begin{array}{l}\text { Power } \\
\text { Factor }\end{array}$ & $\tan \theta$ & $n$ & $v$ & $\epsilon$ & $V\left(R_{1}\right)$ & $V\left(R_{1}\right)$ \\
\hline .95 & .33 & 1.2 & $T$ & .0 .3 & .97 & .72 \\
\hline - & . & 0.8 & 0.4 & -0.2 & 1.07 & .79 \\
\hline .90 & .48 & 1.2 & 1 & -0.3 & .85 & .63 \\
\hline & 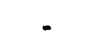 & 0.8 & 0.4 & -0.2 & 1.01 & .72 \\
\hline .85 & .62 & 1.2 & 1 & -0.3 & .76 & .57 \\
\hline • & . & 0.8 & 0.4 & -0.2 & .95 & .67 \\
\hline
\end{tabular}

The value of compensation is greater as $V(R)$ is lower, since these factors discount the difference in generation cost between different sites. Because $\epsilon$ is negative and $\eta>\nu$, compensation at I has less value than at $L$. However, as the power factor of generation decreases, the differences in value diminish somewhat, since VArs are more abundant.

The ranges of values of parameters and generation cost differences that we have presented overlap enough so that cases $A$ and $B$ are each possible for some reasonable combination of values. In either case $A$ or $B$, the interesting qualitative trade-off is then between:

1) bundled production of real and reactive power on the load side of the interface constraint at either I or $\mathrm{L}$, and,

2) transmission or compensation.

This tradeoff depends on the cost of transmission and compensation compared to the difference in the generation costs discounted by the appropriate $V(R)$.

The trade-off with local compensation or transmission raises two particular kinds of cost estimation issues. The compensation alternatives exhibit a wide range of costs depending upon exactly what kind of equipment is used. These choices, in turn, require a much finer specification of the network constraints than those offered here. One source gives cost estimates for compensation that vary from \$10-\$60/kVA (EPRI, 1990). The more expensive alternatives, involving thyristor switching instead of mechanical switches, would be used when some problem in addition to voltage had to be addressed. A smaller range, but a higher absolute value of compensation cost is reported by OFFER $(1992$, pp.74, 77) for the National Grid Company in England. Mechanically switched capacitors are estimated to cost $£ 33 / \mathrm{kVA}(\$ 50 / \mathrm{kVA})$ and static VAr compensators are estimated at $\$ 67 / \mathrm{kVA}(\$ 100 / \mathrm{kVA})$. We expect this cost difference to decrease as power semiconductor technology matures. For high cost compensation devices and 
values of $\eta$ that are substantially below 1 , the effective cost of compensation can exceed $\$ 100 / k W$.

For transmission, costs in the range from $\$ 50-\$ 200 / \mathrm{kW}$ are commonly cited (SCE, 1992). In this case, the range depends critically upon the expected level of utilization of a discrete capacity addition. The lower the level of utilization for a fixed addition, the higher the unit cost. This lumpiness problem, which we have suppressed from our analytical formulation, is fundamental to practical decisions.

Finally, if siting constraints limit the feasibility of transmission construction, our analysis suggests that a number of sub-optimal alternatives will still be available. Furthermore, our method allows estimation of the cost penalty associated with siting constraints.

Our analysis shows that there is a wide range of alternatives available to relieve transmission interface constraints. The cost differences among the alternatives may be small under some conditions. Our method helps to organize and enumerate the choices, clarify what practical conditions dictate the optimum in particular cases, and help to motivate the final choices made by planners. The advantage of a heuristic framework such as ours is that it can help reduce the perception of "black box" planning that often seems to surround transmission issues.

\section{Generalizations and Conclusion}

We have posited a simple linear relationship between interface capacity and the generation and reactive generation on the import side of the interface. The model was verified to be reasonable for the simple three bus system we investigated. It can also be expected to apply approximately to more complex networks that have identifiable remote, intermediate, and local generation, compensation, and load sites. The framework we develop is useful for preliminary comparisons between various options, particularly if optimal power flow software is used to quickly estimate parameters.

Our study indicates that there is considerable potential in analyzing the composite generationtransmission-compensation system when interface constraints are important. Once general tradeoffs have been established, more detailed studies are necessary to quantify costs in detail. The advantage of our analysis is that it incorporates more expansion possibilities than a traditional hierarchical analysis of generation, transmission, and compensation.

Our model can be extended in several ways. First, we do not model upper limits on $P_{L}, P_{l}, P_{G}$; however, there may, in practice, be limits on the possible expansion of generation at L, I or G. This can easily be modeled in the linear programming formulation. Second, percentage real and reactive losses were modeled as constant. In fact, losses vary with loading and will increase significantly as loading increases. A more accurate representation of losses could be incorporated into a non-linear model. Third, the sensitivity factors $\eta, v$ and $\epsilon$ were assumed constant. However, they will vary with loading level, particularly as conditions of voltage collapse are 
approached. Variation of these parameters could also be more accurately represented in a nonlinear model. 


\section{Acknowledgements}

The authors appreciate comments from Thomas Fecho, American Electric Power Service Corporation and Juan Villar, Florida Power and Light Company. This research was funded by the Assistant Secretary for Energy Efficiency and Renewable Energy, Office of Utility Technologies, Office of Energy Management Division of the U.S. Department of Energy under Contract No. DE-AC03-76SF00098. 


\section{References}

Appalachian Power Company (APCo), Case Number PUE910050 Before the State Corporation Commission of Virginia, Appendix, Volume 1, 1991.

Baldick, R. and E. Kahn, Transmission Planning in the Era of Integrated Resource Planning: A Survey of Recent Cases, Report Number LBL-32231, Lawrence Berkeley Laboratory, Berkeley, CA (1992).

Baldick, R. and E. Kahn, "Transmission Planning Issues in a Competitive Economic Environment," IEEE Transactions on Power Systems (1993a).

Baldick, R. and E. Kahn, "Network Costs and the Regulation of Wholesale Competition in Electric Power, "Journal of Regulatory Economics (1993b).

Bloom, et al., "Long-Range Generation Planning Using Generalized Benuers Decomposition: Implementation and Experience," Operations Research vol. 32, no. 2, March, (1984).

Dunlop, R., R. Gutman and P. Marchenko, "Analytical Development of Loadability Characteristics for EHV and UHV Transmission Lines, " IEEE Transactions on Power Apparatus and Systems, vol. PAS-98, no.2 (1979) 606-617.

EPRI, "Electric Generation Expansion Analysis System (EGEAS)," Report EL-2561, prepared by Massachusetts Institute of Technology, (1982a).

EPRI, "Transmission System Reliability Methods, " Vol. 1, Report EL-2526, prepared by Power Technologies, Inc., (1982b).

EPRI, "An Approach for Determining Transfer Capability Objectives," Vol. 1, Report EL-3425, prepared by Power Technologies, Inc., March, (1984a).

EPRI, "Optimization of Reactive Volt-Ampere (VAr) Sources in Sy tem Planning," Vol. 1, Report EL-3729, prepared by Scientific Systems, Inc., (1984b).

EPRI, "Composite Generation-Transmission Expansion Planning," Report EL-5179, prepared by Centro De Pesquisas de Energia Eletrica, June, (1987).

EPRI, "Mathematical Decomposition Techniques for power System Expansion Planning," Vol 1: Decomposition Methods and Uses, Report EL-5299, prepared by Stanford University, February, (1988a).

EPRI, "Mathematical Decomposition Techniques for power System Expansion Planning," Vol 3: An Integrated Methodology for VAr Sources Planning, Report EL-5299, prepared by Stanford University, February, (1988b).

EPRI, "Technical Limits to Transmission System Operation," Report EL-5859, prepared by Power Technologies, Inc., June, (1988c).

EPRI, "Flexible ac Transmission Systems (FACTS): Scoping Study," Vol. 1, Part 1: Analytical Studies, Report EL-6943, prepared by Power Technologies, Inc., August, (1990). 
EPRI, "Flexible ac Transmission Systems (FACTS): Scoping Study," Vol. 2, Part 1: Analytical Studies, Report EL-6943, prepared by General Electric Company, September, (1991).

Gonen, T. Electric Power Transmission System Engineering, John Wiley and Sons, Inc., New York NY, 1989.

Head, W. J. et al., "The Procedure Used to Assess the Long Range Generation and Transmission Resources in the Mid-Continent Area Power Pool," IEEE Transactions on Power Systems, vol. 5, no. 4, (1990) 1137.1145.

Hong, Y-Y. et al., "Multi-Year Multi-Case Optimal VAr Planning," IEEE Transactions on Power Systems, vol. 5, no. 4, (1990) 1294-1301.

Hopewell Cogeneration Inc., Amendment No. 1 to Power Purchase and Operating Agreement Between Hopewell Cogeneration Inc. and Virginia Electric and Power Corporation, 1988.

IEEE, "Reliability Assessment of Composite Generation and Transmission Systems," Tutorial Course Text 90EH0311-1-PWR, (1990).

Kahn, E., "Regulation by Simulation: The Role of Production Cost Models in Electricity Planning and Pricing," Presented at Rutgers University Advanced Workshop in Regulation and Public Utilities Economics, 6th Annual Western Conference, Monterey, CA, June, (1993).

Lebow, et al., "A Hierarchical Approach to Reactive Volt-Ampere (VAr) Optimization in System Planning," IEEE Transactions on Power Apparatus and Systems, vol. PAS-104, no. 8, August, (1985) 2051-2057.

Mallard, S., Staff Testimony in Case No. PUE910050 before the State Corporation Commission of Virginia, 1992.

Office of Electricity Regulation (OFFER), Report on Constrained-On Plant, 1992.

Opoku, G. "Optimal Power System VAr Planning," IEEE Transactions on Power Systems, vol. 5, no. 1 (1990) $53-60$.

Rogers, J. and M. Rolko, "A Quadratic Programming Model for Planning Generation and Inter-Utility Transmission," Electrical Power and Energy Systems, v.14. no.1 (1992) 18-22.

Southern California Edison (SCE), Transmission Cost Tables, 1992.

Staschus, K. et al., "A Multi-Attribute Evaluation Framework for Electric Resource Acquisition in California," Electrical Power and Energy Systems, v.13. no.2 (1991) 73-80.

Stoll, H. Least Cost Electric Utility Planning, John Wiley and Sons, Inc., New York NY, 1989.

Wenyuan, L. and R. Billinton, "A Minimum Cost Assessment Method For Composite Generation and Transmission System Expansion Planning," IEEE Transactions on Power Systems, v.8, no.2 (1993) 628-635. 


\section{Appendix A}

\section{A.1 Proof of Fact}

First notice that, in case $A$, it is never optimal to build generation at $G$ and $L$ in preference to generation at $\mathbf{G}$ and $\mathrm{I}$. The choices are therefore between generation at $\mathrm{G}$ and $\mathrm{I}$ and generation at $\mathbf{G}$ and either compensation or transmission. The stated conditions compare the relative prices of these options. In case $B$, it is never optimal to build generation at $G$ and $I$ in preference to generation at $\mathrm{G}$ and $\mathrm{L}$. The optimal construction involves generation at $\mathrm{G}$, with choices between local generation, local compensation, and transmission. The conditions compare the relative prices of these options. The result in the case of a limit on the increase in interface constraint follows because the optimization formulation is linear. 
